\title{
Contrefaire, subvertir. Portrait de Frobenius / Shrobénius dans Le Devoir de violence de Yambo Ouologuem
}

Éléonore Devevey

\section{(2) OpenEdition}

Édition électronique

URL : https://journals.openedition.org/coma/3953

DOI : 10.4000/coma.3953

ISSN : 2275-1742

Éditeur

Institut des textes \& manuscrits modernes (ITEM)

Référence électronique

Éléonore Devevey, «Contrefaire, subvertir. Portrait de Frobenius / Shrobénius dans Le Devoir de violence de Yambo Ouologuem », Continents manuscrits [En ligne], 12 | 2019, mis en ligne le 09 mai 2019, consulté le 13 janvier 2023. URL : http://journals.openedition.org/coma/3953

Ce document a été généré automatiquement le 13 janvier 2023.

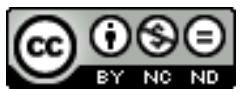

Creative Commons - Attribution - Pas d'Utilisation Commerciale - Pas de Modification 4.0 International - CC BY-NC-ND 4.0

https://creativecommons.org/licenses/by-nc-nd/4.0/ 


\title{
Contrefaire, subvertir. Portrait de Frobenius / Shrobénius dans Le Devoir de violence de Yambo Ouologuem
}

\author{
Éléonore Devevey
}

... sous cette masse gigantesque d'œuvres des maîtres à penser qui puisent leur matière dans les archives du sang, il faut, par le jeu du langage, se faire le raconteur d'une incomparable série dont la qualité (et les motivations ainsi justifiées) ne se démentent pas.

Yambo Ouologuem, Lettres à la France nègre

L'implantation de l'ethnologie dans le paysage intellectuel français, au cours $\mathrm{du} \mathrm{xx}^{\mathrm{e}}$ siècle, n'a pas seulement influé sur la façon dont les écrivains définissent leurs démarches et leurs ambitions. Elle a aussi eu pour effet secondaire l'entrée en fiction de la figure de l'ethnologue : lignée qui va du folkloriste Dussouchel, dans Les Temps mêlés et Saint-Glinglin de Raymond Queneau, jusqu'aux personnages d'ethnographes chez Patrick Chamoiseau, en passant par Marcel Appenzzell, l'« anthropologue incompris » de La Vie mode d'emploi. On aurait tort de voir dans ces avatars fictionnels de simples curiosités incidentes : ils sont une façon de prendre acte de la pression qu'exercent les savoirs constitués sur l'invention littéraire, et le témoin des enthousiasmes et des doutes que ces savoirs ont pu éveiller chez les écrivains ${ }^{1}$. Les enjeux de cette représentation fictionnelle sont tout particulièrement vifs en situation post-coloniale, dans la mesure où l'héritage ethnologique est une composante aussi essentielle que controversée de la définition de l'auctorialité post-coloniale ${ }^{2}$. Que les personnages d'ethnologue soient dans ce cadre un moyen de mettre en question l'autorité de la science (néo-)coloniale semble s'imposer : difficile de ne pas voir en eux, suivant la voie jadis ouverte par Julius Lips ${ }^{3}$, une façon de «riposter » à l'ordre colonial du savoir. 
Moins mécaniquement, j'aimerais y voir un effort pour le défaire, en soi et dans le texte - et pour complexifier l'axiologie de la relation ethnographique.

Le personnage de Fritz Shrobénius, ethnologue de passage dans l'empire ouest-africain imaginaire du Nakem, dont Le Devoir de violence de Yambo Ouologuem $(1968)^{4}$ est la chronique sanguinaire, permet d'éprouver cette hypothèse. Instrument d'une satire féroce, du savoir occidental comme de la «collaboration" africaine qui le rend possible, ce personnage constitue une façon de décoloniser, en les carnavalisant, les représentations de l'histoire africaine. Premier roman publié par Ouologuem (et unique roman publié sous son nom), Le Devoir de violence raconte l'histoire de la dynastie tyrannique des Saiff, qui règne depuis le XIII ${ }^{\mathrm{e}}$ siècle sur le Nakem en opprimant la « négraille »; il retrace aussi l'histoire de Raymond-Spartacus Kassoumi, fils d'esclaves de Saiff, qui, parti étudier en France, deviendra en 1947 député de l'Union française. Résolument anti-réaliste et démystificateur, le roman met en avant la violence de l'Afrique pré-coloniale, la collaboration machiavélique des notables africains à l'ordre colonial, et le passage des masses d'un joug à l'autre : le parcours de Kassoumi, à ce titre, fait partie de «l'œuvre de soumission de la négraille instruite» (DV, p. 191) fomentée par Saif. Tableau de corruption généralisée, doublé d'une veine érotique et fondé sur une conception cyclique de l'Histoire, cette chronique épique a ceci de paradoxal qu'elle est foncièrement ironique, et son axiologie constamment ambiguë sa réception, complexe, et les accusations de plagiat portées contre l'auteur, témoignent de ce qu'un tel texte a pu avoir de déroutant ${ }^{5}$.

Dans les chapitres 4 et 5 de la partie centrale du roman, intitulée "La Nuit des géants ", apparaît le personnage de Fritz Shrobénius, savant bouffon dont le nom est démarqué de celui de l'ethnologue allemand Leo Frobenius (1873-1938). À travers ce personnage secondaire, Ouologuem incrimine le primitivisme mercantile qui se met en scène sous des dehors scientifiques. Mais il marque aussi ses distances, par la relecture critique qu'il engage de l'œuvre de Frobenius, avec les partis anthropologiques des écrivains de la négritude. Façon, en somme, de faire voler en éclats, par les moyens de la fiction et les ressources de l'intertextualité, l'idée d'un sens unique - direction et signification - de la relation ethnographique.

\section{Fritz Shrobénius : " ethnologue rusé », « marchand- confecteur d'idéologies »}

4 Le but premier de la venue au Nakem de Fritz Shrobénius, située en 1910, est l'acquisition d'artefacts locaux. Prodigue en cadeaux envers Saiff et son peuple, l'ethnologue s'essaye aussi à un travail de collecte d'informations et de mythes. Ouologuem fait de lui l'incarnation d'un primitivisme frénétique, sinon pathologique :

[...] écrevisse humaine frappée de la manie tâtonnante de vouloir ressusciter, sous couleur d'autonomie culturelle, un univers africain qui ne correspondait à plus rien de vivant ; habillé avec une élégance tapageuse de colon en fête, riant souvent, il voulait trouver un sens métaphysique à tout, jusques à la forme de l'arbre à palabres où devisaient les notables. Gesticulant à tout propos, il étalait son " amitié » pour l'Afrique et son savoir orageux avec une assurance de bachelier repêché. Il considérait que la vie africaine était art pur, symbolisme effroyablement religieux, civilisation jadis grandiose - hélas victime des vicissitudes de l'homme blanc - puis, sitôt qu'il lui fallait constater 
l'aridité spirituelle de certaines manifestations de la vie sociale, il tombait dans une sorte de somnolence hébétée, étant même incapable de tristesse. (DV, p. 102)

5 Le personnage concentre les travers dont sera taxée (une bonne partie de) l'ethnologie africaniste (des années 1930 aux années 1960): fascination de l'antérieur et de l'authentique, à l'origine d'un savoir embaumant ; manie interprétative qui s'exerce au mépris du bon sens ; mélange de naïveté philanthropique et d'arrogance de dominant ; besoin forcené de voir dans l'Afrique l'envers édénique du monde occidental - et les ébats de sa fille avec le prince Madoubo actualisent le versant érotique de cette même voracité à l'égard de la «négraille ». Si le patronyme du personnage (la substitution de Fritz à Leo, évacuant le lion, accuse la germanité du personnage, tout comme le prénom de son épouse Hildegaard) et ses théories sont démarqués de ceux de Frobenius (nous y reviendrons), c'est sans doute Marcel Griaule qu'a en tête Ouloguem lorsqu'il brosse ce portrait ${ }^{6}$ : on reconnaît les méthodes, martiales, aussi bien que les désirs sous-jacents à l'entreprise savante - et l'on peut sans peine retrouver les excès interprétatifs de Shrobénius en Dieu d'eau, dont la cohérence cosmologique est tellement parfaite qu'elle en devient suspecte ${ }^{7}$. L'hypothèse est d'autant plus vraisemblable que Ouologuem est né en 1940 en pays dogon ${ }^{8}$, «terrain phare de l'ethnologie française puis de l'école Griaule " , où ce dernier a pour informateur et interprète un certain Dousso Wologuem ${ }^{10}$ : les terrains de Griaule font donc vraisemblablement partie du patrimoine mémoriel de l'écrivain. Shrobénius, "marchand-confectionneur d'idéologie » habile à faire fonctionner les "tiroirs-caisses » (DV, p. 111) de tous les musées du monde (on songe à l'entregent de Griaule pour le financement de ses missions), incarne quoi qu'il en soit une exaltation à la fois naïve, cynique et prédatrice de l'Afrique qui sera reprochée aux grandes « missions » ethnographiques de l'entre-deux-guerres. Sa visite au Nakem a pour effet d'enclencher les spéculations intellectuelles et financières sur " l'art nègre », dont Saïf lui-même fait le jeu. Shrobénius a en effet

[...] l'intuition lunatiquement géniale de clamer, parlant du Nakem, de sa civilisation et de son passé : « Mais ces gens sont policés, civilisés jusqu'aux os! Partout, avenues larges, calmes, paisibles, où l'on respire la grandeur d'un peuple, son génie humain... Il a fallu que l'impérialisme blanc s'infiltrât là, avec sa violence, son matérialisme colonisateur, pour que ce peuple si civilisé, brusquement dégringolât à l'état sauvage, se vît taxé de cannibalisme, de primitivisme, alors qu'au contraire - témoin : la splendeur de son art - la grandeur des empires du Moyen Âge constituait le vrai visage de l'Afrique, sage, belle, riche, ordonnée, non violente et puissante tout autant qu'humaniste - berceau même de la civilisation égyptienne. (DV, p. 111)

6 Ouologuem pastiche ici Frobenius : l'éloge de la civilisation africaine placé dans la bouche de Shrobénius est directement décalqué d'une célèbre page de l'ethnologue (quand l'Afrique « berceau de la civilisation égyptienne » est une allusion aux théories de Cheikh Anta Diop $\left.{ }^{11}\right)$. En effet, dans la deuxième section du «livre premier » de son Histoire de la civilisation africaine, intitulée "Que signifie pour nous l'Afrique?", Frobenius, prenant le contre-pied de la conception hégélienne de l'Afrique développée dans La Philosophie de l'histoire, s'essayait à reconstituer le regard des premiers colons sur le continent. Celui-ci dut leur apparaître, estime-t-il, comme un modèle d'harmonie et d'opulence :

Lorsqu'ils arrivèrent dans la baie de Guinée et abordèrent à Vaïda, les capitaines furent fort étonnés de trouver des rues bien aménagées, bordées sur une longueur de plusieurs lieues par deux rangées d'arbres ; ils traversèrent pendant de longs jours une 
campagne couverte de champs magnifiques, habités par des hommes vêtus de costumes éclatants dont ils avaient tissé l'étoffe eux-mêmes ! Plus au sud, dans le Royaume du Congo, une foule grouillante habillée de "soie » et de « velours », de grands États bien ordonnés, et cela dans les moindres détails, des souverains puissants, des industries opulentes. Civilisés jusqu'à la moelle des os! Et toute semblable était la condition des pays à la côte orientale, la Mozambique, par exemple.

Les révélations des navigateurs $d u \mathrm{XV}^{\mathrm{e}}$ au XVII ${ }^{\mathrm{e}}$ siècle fournissent la preuve certaine que l'Afrique Nègre qui s'étendait au sud de la zone désertique du Sahara était encore en plein épanouissement, dans tout l'éclat de civilisations harmonieuses et bien formées. Cette floraison, les conquistadores européens l'anéantissaient à mesure qu'ils progressaient. Car le nouveau pays d'Amérique avait besoin d'esclaves et l'Afrique en offrait : des centaines, des milliers, de pleines cargaisons d'esclaves! Cependant la traite des Noirs ne fut jamais une affaire de tout repos ; elle exigeait sa justification ; aussi fit-on du Nègre un demi-animal, une marchandise. Et c'est ainsi que l'on inventa la notion du fétiche (portugais : feticeiro) comme symbole d'une religion africaine. Marque de fabrique européenne ! Quant à moi, je n'ai vu dans aucune partie de l'Afrique Nègre les indigènes adorer des fétiches.

L'idée du « Nègre barbare » est une invention européenne qui a, par contrecoup, dominé l'Europe jusqu'au début de ce siècle ${ }^{12}$.

7 Le propos de Frobenius est, plus largement, de mettre en évidence l'unité civilisationnelle et stylistique de l'Afrique noire, civilisation où tout est art et poésie. Ouologuem condense donc ici le propos de Frobenius, en en conservant l'esprit. Shrobénius a, comme son précédent historique, le désir d'inverser le scénario de l'histoire officielle de la colonisation: l'Afrique, avec elle, ne serait pas passée de la sauvagerie à la civilisation, mais de l'épanouissement à la déchéance. Que ce discours, dans la fiction, soit attribué à un personnage ridicule et cupide en fait un simple retour de balancier, aussi indigent que les conceptions qu'il prend à rebours.

\section{Travail de sape par ricochet}

8 Ces lignes fameuses de Frobenius ont essaimé au cours du demi-siècle qui a suivi leur rédaction: en les invoquant, Ouologuem ne convoque pas seulement leur auteur, mais aussi ceux qui les ont reprises avant lui. Pastichant Frobenius, c'est également l'héritage de la négritude que Ouologuem prend pour cible: ses fondateurs avaient pour leur part trouvé dans les écrits de Frobenius de quoi étayer leurs propres théories et revendications ${ }^{13}$. Un grand nombre des publications clés du mouvement ont de fait donné à lire les travaux de l'ethnologue allemand. La Revue du monde noir, en 1932, a d'abord publié des pages traduites en français, sous le titre "Le spiritisme dans l'intérieur de l'Afrique "; l'ethnologue était alors présenté comme celui qui « fait surgir l'Afrique de la nuit des temps, avec ses traditions et sa culture passées ${ }^{14} »$. Dans le $n^{\circ} 5$ de Tropiques ${ }^{15}$, Aimé Césaire publie les pages qui seront pastichées par Ouologuem, et les convoquera à nouveau dans le Discours sur le colonialisme, lorsqu'il amorce, à la fin de sa troisième partie, une "apologie de nos vieilles civilisations nègres: c'étaient des civilisations courtoises ", et de ceux qui l'ont perçu :

Et de ce qu'ont dit, de ce qu'ont vu les premiers explorateurs [parlons-en]... Pas de ceux qui mangent aux râteliers des Compagnies! Mais des d'Elbée, des Marchais, des Pigafetta! Et puis de Frobenius! Hein, vous savez qui c'est, Frobenius? Et nous lisons 
ensemble : «Civilisés jusqu'à la mœlle des os ! L'idée du Nègre barbare est une invention européenne $»^{16}$.

9 La position de Césaire est toutefois ambiguë sur ce point car à cet l'éloge (assez indirect) de Frobenius fait immédiatement suite une dénonciation des « ethnographes métaphysiciens et dogonneux ", "tous tenants déclarés ou honteux du colonialisme pillard $^{17} »:$ l'œuvre de Frobenius semble déjà conçue par Césaire comme une étape à dépasser. Au même moment, Léon-Gontran Damas emprunte aux travaux de Frobenius des gravures rupestres pour accompagner ses Poèmes nègres sur des airs africains ${ }^{18}$. Pour Senghor, qui l'a découvert par Césaire et lui a consacré de nombreux textes, sa lecture eut l'effet d'« un coup de tonnerre » : «nul mieux que Frobenius ne révéla l'Afrique au monde et les Africains à eux-mêmes ». Et, un peu plus loin :

C'est Leo Frobenius qui nous donna, et la vision, et l'explication [philosophique des valeurs noires] au moment même où, les études terminées, nous entrions dans la vie active, militante, le mot et l'idée de la Négritude dans notre gibecière. C'est Frobenius qui nous aida à charger le mot de sa signification la plus dense, la plus humaine en même temps ${ }^{19}$.

10 Frobenius est à l'évidence l'argument d'autorité ethnologique de la génération de la négritude - et ces mêmes lignes de Frobenius sont aussi citées par ses relais français, André Breton dans "Un grand poète noir", sa préface à Cahier d'un retour au pays natal ${ }^{20}$, et Georges Balandier dans Afrique ambiguë, en $1957^{21}$. Mais pour la génération qui vient à l'écriture après les indépendances, prendre appui sur ses travaux ne constitue plus une position tenable : l'urgence est alors de se défaire des biais anthropologiques des inventeurs de la négritude. Dans Le Devoir de violence, les productions inspirées de Frobenius sont taxées, par allusion, de "romantisme nègre » (DV, p. 14), remisées dans le grand fourre-tout de « la littérature shrobéniusologique salivant, en un rusé mélange de mercantilisme et d'idéologie, la splendeur de la civilisation nègre" (DV, p. 189). Ouologuem fait ainsi apparaître, par l'oblique et dans la fiction, l'inscription coloniale de la négritude - propos qui entre en résonance avec la critique de Frantz Fanon de l'ontologisme senghorien, formulée dans Peau noire, masques blancs (1952), ou celles, postérieures, de Paulin Hountondji (rassemblées dans Sur la "philosophie» africaine, 1977) ou encore de Stanislas Adotevi, dans Négritude et négrologues (1972 ${ }^{22}$.

11 Cette position, Ouologuem l'a explicitée dans ses Lettres à la France nègre, parues en 1969, un an après Le Devoir de violence, et principalement dirigées contre ce qu'il appelle la «négrophilie philistine ». Ouologuem estime qu'il est devenu « impossible de fermer les yeux devant l'image d'une Afrique par trop déformée par ses chantres et ses littérateurs ", et invite à se défaire d'une " attitude d'esclave - qui consiste à toujours se définir, non par rapport à soi et à une réalité véritablement africaine, mais eu égard au Blanc et aux canons de civilisations reconnus par ce dernier ». L'héritage de la négritude est alors considéré avec plus de nuance :

Si la négritude, cependant, vaut toujours parce qu'elle est un cadre auquel il reste encore à donner meilleur contenu, ce contenu ne saurait être que s'il n'érige pas des autels et des statues à cent mythes, qui ne répondent et n'ont jamais correspondu à quoi que ce soit de vivant en Afrique : foire aux chimères où s'est exaltée l'imagination de plus d'un marchand d'idéologie, échafaudant mille impostures dont le mérite - peutêtre - est de rassurer, à la Bourse des valeurs de la primitivité, tous les petits rentiers de la tragi-comédie...

En vérité, ceux-là qui (journalistes, sociologues, ethnologues, africanistes, littérateurs 
et négrophiles « spécialisés ») chercheraient à rêver l'Afrique à la mesure de leur bonne conscience, je les soupçonne de vouloir - sous le prétexte fallacieux de la servir l'encapsuler dans le génie de la littéralitéén.

Pour Ouologuem, la négritude "vaut » désormais comme point d'appui à dépasser, et non plus comme ligne de pensée ou d'action : trahissant la réalité africaine, les mythes qui la sous-tendent et qu'elle a pu favoriser ont à ses yeux pour motif ultime la recherche du profit, symbolique ou financier.

\section{La relation ethnographique : affaire de faussaires?}

Écrivant Le Devoir de violence, Ouologuem entendait vraisemblablement purger les représentations de l'Afrique de toute trace de manichéisme: ni noir ni blanc en l'occurrence. Le ressort de ce grand nettoyage est de faire du cynisme et de la cupidité les choses du monde les mieux partagées. L'attitude de l'ethnologue comme de son informateur, tous deux également fabulateurs, agissant également au mépris l'un de l'autre, consiste ainsi à se rendre mythe pour mythe, fausse-monnaie pour faussemonnaie. Si l'ethnologue, « sécrétant son propre mythe, [...] soucieux de sa publicité mais se gaussant d'une société qui lui avait tout donné » (DV, p. 111), exploite sans vergogne ses enquêtés, ceux-ci ont su en amont devancer les attentes de l'ethnologue, sans être dupes du rôle qu'il leur donnait ${ }^{24}$. Ouologuem épingle de la sorte une relation de dépendance réciproque : de ses théories,

[...] Shrobénius, de retour au bercail, [...] tira un double profit : d'une part, il mystifia son pays, qui, enchanté, le jucha sur une haute chair sorbonicale, et, d'autre part, il exploita la sentimentalité négrillarde - par trop heureuse de s'entendre dire par un Blanc que «l'Afrique était ventre du monde et berceau de civilisation ». La négraille offrit par tonnes, conséquemment et gratis, masques et trésors artistiques aux acolytes de la «shrobéniusologie ». (DV, p. 111)

$14 \mathrm{Au}$ "double profit » de Shrobénius qui, par l'exaltation de l'Afrique promise à faire école ${ }^{25}$, a trouvé le moyen d'accroître son propre capital symbolique et d'enrichir ses collections, répond la «collaboration » africaine, elle-même double : active et cynique, par appât du gain, de la part des élites; passive et aliénée, de la part du peuple, trop accessible à ce qui flatte son orgueil. Cette logique de dépendance et de tromperie réciproques est aussi à l'œuvre dans le tour de passe-passe de Saif, qui, devant la pénurie d'artefacts locaux à écouler à Shrobénius, fait "enterrer des quintaux de masques hâtivement exécutés à la ressemblance des originaux ", puis les exhume pour les revendre à prix d'or, "masques vieux de trois ans, chargés, disait-on, $d u$ poids de quatre siècles de civilisation » (DV, p. 112), répondant ainsi à l'imposture (intellectuelle) par l'imposture (artisanale). On peut voir, dans ce jeu de contrefaçons croisées, une image à la fois de l'ambition critique de Ouologuem, mais aussi de la poétique : leur visée commune est de saper toutes les sources d'autorité et les marqueurs d'identité stables $^{26}$.

15 Le pastiche stratifié de Frobenius, par lequel l'écrivain ouvre le dialogue non seulement avec la tradition ethnologique, mais aussi avec ses aînés, montre en effet que la pratique d'emprunts textuels de Ouologuem relève d'un art consommé du dialogisme intertextuel, et non d'un acte de plagiat inconscient de lui-même, ou destiné à passer inaperçu: il suffit, pour s'en convaincre, de lire la démonstration de "gymnastique 
opératoire de l'écriture » qu'il donne dans ses Lettres à la France nègre, rapprochant ses méthodes d'écriture de celles d'un Raymond Roussel ou de l'OuLiPo ${ }^{27}$. Se défendant, dans une lettre adressée à Paul Flamand, directeur du Seuil, et datée du 15 mars 1970, de l'accusation d'avoir plagié l'écrivain américain John Mac Donald, Ouologuem mentionnait en bonne place Frobenius / Shrobénius :

[...] John Mac Donald est cité, comme Ki Zerbo, comme Leo Frobenius (sous le nom de Schrobénius) comme Okba ben Nafi el Fitri, comme le Tarik, divers griots, chroniqueurs noirs, s'exprimant en langues vernaculaires regroupant les principaux groupes linguistiques africains (cf les italiques du roman) comme divers historiens arabes, portugais, espagnols, coptes, éthiopiens, ou, plus généralement, dans une perspective d'universalité du conflit violence et non violence, la Bible et le Coran, la correspondance de missionnaires blancs, Tacite, Suétone, Schwarz Bart (puisque l'Éthiopie, par Haïlé Sélassié juif de par la reine de Saba, ouvre la dimension de la conscience malheureuse antérieure à la négritude, et, dès lors, affranchie de la dialectique usée Noirs-Blancs), et enfin, deux maîtres du réalisme désabusé : Flaubert et Maupassant. Mon propos, ce faisant, était davantage l'histoire légendaire, puisque c'est par la seule légende qu'il est possible de créer un discours à l'intérieur de mythe vaste des civilisations en devenir. L'architecture initiale de mon roman était autre, en clins d'œil, références, guillemets, narrations, analyses ${ }^{28}$.

L'écriture de Ouologuem est intrinsèquement intertextuelle ${ }^{29}$ et ironique ${ }^{30}$ : le dispositif d'emprunts textuels non signalés qui sous-tend le roman brouille toutes les sources d'autorité intellectuelle, de même que le récit ruine toutes les formes d'autorité morale ${ }^{31}$. La poétique de ce texte composite vise aussi, du même coup, à contrer toute conception essentialiste de l'identité. Le contraste est, de fait, maximal entre Les Fondements de l'africanité ${ }^{32}$, selon Senghor, paru un an avant Le Devoir de violence - et dont la première section, intitulée "Afrique berceau de l'humanité », propose une synthèse des thèses des préhistoriens et des anthropologues à ce sujet et postule l'unité de la civilisation africaine - et le roman, qui met en relief les violences précoloniales et les guerres intestines, et jette le doute sur l'authenticité de toutes les traditions, indigènes ou étrangères. Cet effort de dissolution identitaire apparaît aussi bien dans la manipulation des ethnonymes, pointée par Thomas Hale - si les Peuhl gardent leur nom, les Mandé deviennent les Randé, et les Dogons, par anagramme, les Ngodos, etc. ${ }^{33}$ - que dans le destin de Kassoumi, anti-héros, intellectuel aliéné, dont l'exil en France est une expérience de perdition et de désubjectivation ${ }^{34}$. Mais si, lors de la parution du Devoir de violence, l'heure était à la mort de l'auteur, à la dissolution du sujet, à l'intertextualité et à la combinatoire, ce n'est pas à ces prismes qu'a d'abord été lu le roman de Yambo Ouologuem.

«Certains transmettent les choses en les rendant intangibles et en les conservant; d'autres transmettent les situations en les rendant maniables et en les liquidant. Ce sont ces derniers que l'on appelle les destructeurs ", écrivait Walter Benjamin dans un texte de $1931^{35}$. En convoquant sauvagement Leo Frobenius et, à travers lui, ceux à qui il a servi d'argument d'autorité, Ouologuem s'illustre comme " caractère destructeur » de premier plan. Le personnage de Fritz Shrobénius, moyen d'incriminer la relation ethnographique en contexte colonial et de prendre ses distances avec l'essentialisme de la négritude senghorienne, apparait, plus fondamentalement encore, comme une manière de ruiner le mythe de l'authenticité, de la relation unilatérale et univoque entre original et copie, entre mystifiés et mystificateurs - ce que Bachir Diagne rendait 
avec humour, dans " 1968 : crisis in African letters »: «to celebrate the novel as the first true African narrative is the same as claiming to have found an authentic African artifact in a shop at the airport $»^{36}$. L'écriture est pour Ouologuem travail de sape contre la possibilité d'établir les sources, de distribuer les rôles et d'arrêter les valeurs, dans la fabrique textuelle comme dans le tourniquet de la relation ethnographique : façon de contrefaire toutes les formes d'autorité pour mieux défaire leur empire. Offrant de nouvelles impressions d'Afrique, Le Devoir de violence délivre ainsi un portrait de l'écrivain en faussaire, plus qu'en ethnologue - faussaire qui, en l'occurrence, fait un feu de joie de ses notions d'ethnologie.

\section{NOTES}

1. Je suis ici le propos de Nathalie Piégay, dans L'Érudition imaginaire, Droz, Genève, 2009 : "L'érudition imaginaire ne caractérise pas une littérature savante : elle traduit la pression que l'accumulation des discours et des savoirs exerce sur l'invention. L'érudition est le miroir sur lequel se projettent les doutes de la littérature : doutes sur ses pouvoirs de représentation, sur sa relation à l'histoire et aux savoirs, sur sa capacité à transmettre ces traces du passé sans lesquelles elle semble ne pas pouvoir se penser» (p. 57). Le personnage du savant fait partie de la topique déterminée par l'implantation de l'érudition dans la fiction. À ce sujet, voir Jacques Noiray, « Figures du savant », Romantisme, n 100, vol. 28, 1998, p. 143-158.

2. Voir par exemple Christopher Miller, Theories of Africans: Francophone Literature and Anthropology in Africa, Chicago, University of Chicago Press, 1990 ; Anna Lesne, «La fabrique des identités aux Antilles "françaises". Discours savants, discours littéraires, rayons de bibliothèque ", thèse de doctorat, sous la direction de Jean-Luc Bonniol, Aix-Marseille, Université de Provence, 2011; Vincent Debaene, «Les écrivains contre l'ethnologie? Ethnographie, ethnologie et littérature d'Afrique et des Antilles, 1921-1948», Romanic Review, 104, 3-4, 2013, p. 353-374.

3. Julius Lips, The Savage hits back or The White Man Through Native Eyes, Yale University Press, 1937 ; avec une préface de Malinowski. Dans cet ouvrage polémique, l'ethnologue allemand, exilé aux États-Unis après avoir été chassé de son poste par le régime nazi, se donnait pour objectif de reconstruire le point de vue du «sauvage» sur le «blanc». Si l'approche est alors novatrice, l'argumentation est toutefois biaisée : Lips voulait voir dans toutes les représentations de blancs des «ripostes» contre le colonisateur, des caricatures féroces, ce qui négligeait parfois la complexité de telles représentations.

4. Yambo Ouologuem, Le Devoir de violence, Paris, Seuil, 1968. Désormais abrégé DV.

5. D'abord accueilli avec enthousiasme (prix Renaudot en 1968), puis accusé de plagiat, soupçonné de donner une représentation faussée de l'histoire africaine, le roman a longtemps été épuisé, provoquant la mort symbolique de son auteur. Ce n'est qu'en 2002 qu'il a été réédité en France, au Serpent à plumes, puis en 2018, dans la collection «Cadre rouge » du Seuil, où il était initialement paru. Voir à ce sujet l'étude de Jean-Pierre Orban, "Livre culte, livre maudit: Histoire du Devoir de violence de Yambo Ouologuem », Continents manuscrits [en ligne], mai 2018. URL : http://journals.openedition.org/coma/1189 (page consultée le 26 mars 2019). Sur les emprunts textuels, les sources et la véracité de la représentation de l'histoire africaine qu'il propose, voir notamment Thomas Hale, «Challenging the Past: A Modern Interpretation of the 
Songhay Empire in Yambo Ouologuem's Le Devoir de violence ", chapitre de son ouvrage Scribe, Griot and Novelist, Narrative Interpreters of the Songhay Empire, University of Florida Press, 1990, p. 135-159.

6. Cette piste est évoquée par Vincent Debaene, dans L'Adieu au voyage. L'ethnologie française entre science et littérature, Paris, Gallimard, coll. « Bibliothèque des idées », 2010, p. 435.

7. Marcel Griaule, Dieu d'eau. Entretiens avec Ogotemmêli, Paris, Éditions du Chêne, 1948. On trouve également dans le roman une allusion à L'Afrique fantôme (DV, p. 22).

8. Pour plus de précisions biographiques sur Ouologuem, voir la préface de Christopher Wise, dans la réédition de 2003, p. 7-20: Wise évoque le contexte culturel, religieux et politique dans lequel l'auteur a grandi, et les rapports complexes à l'islam comme à la colonisation française que ce contexte conditionne.

9. Éric Jolly, «Démasquer la société dogon. Sahara-Soudan (janvier-avril 1935)», Les Carnets de Bérose, $\mathrm{n}^{\circ} 4$, Lahic / DPRPS-Direction générale des patrimoines, 2014, p. 8.

10. Ibid., notes 15 et 16. Éric Jolly précise que l'orthographe du patronyme varie d'une occurrence à l'autre. Ancien combattant décoré, naturalisé français, Dousso Wologuem est traité et honoré comme un alter ego par les enquêteurs occidentaux. Voir également la notice biographique qui lui est consacrée dans le projet «À la naissance de l'ethnologie française. Les missions ethnographiques en Afrique subsaharienne (1928-1939)», 2016, URL: http:// naissanceethnologie.fr/files/pdf/81.pdf (page consultée le 26 mars 2019).

11. Cheikh Anta Diop, Nations nègres et Culture. De l'antiquité nègre égyptienne aux problèmes culturels de l'Afrique noire d'aujourd'hui, Paris, Présence Africaine, 1955.

12. Leo Frobenius, Histoire de la civilisation africaine [1933], traduit de l'allemand par Dr H. Back et D. Ermont, Paris, Gallimard, [1936] 1952, p. 16-17.

13. Christopher Miller, Theories of Africans: Francophone Literature and Anthropology in Africa, op. cit., p. 16-21. Du même, voir également, «Trait d'union: Injunction and Dismemberment in Yambo Ouologuem's Le Devoir de violence ", L'Esprit créateur, vol. 23, n 4, 1983, p. 62-73.

14. La Revue du monde noir, reproduction en fac-similé, Paris, Jean-Michel Place, 1992, p. 275-280, p. 276.

15. «Que signifie pour nous l'Afrique?», Tropiques, $n^{\circ} 5$, avril 1942 (Tropiques, 1941-1945, reproduction en fac-similé, Paris, Jean-Michel Place, 1994). Dès le premier numéro de la revue, Suzanne Césaire avait signé un texte intitulé «Léo Frobenius et le problème de la civilisation ». Dans l'« Entretien avec Aimé Césaire par Jacqueline Leiner» qui accompagne la réédition de Tropiques (p. V-XXIV), Césaire explique qu'il connaissait, étudiant, « un peu d'ethnographie, grâce à Senghor, d'ailleurs. Cette ethnographie était contenue tout entière dans le nom de Frobenius et de son ouvrage : Histoire de la civilisation africaine » (p. VIII-IX).

16. «Discours sur le colonialisme» [1948], repris dans Écrits politiques, 1935-1956, Paris, JeanMichel Place, 2013, p. 303-329, p. 314-315.

17. Ibid.

18. Pointé par Vincent Debaene, «Les écrivains contre l'ethnologie ? Ethnographie, ethnologie et littérature d'Afrique et des Antilles, 1921-1948», op. cit., p. 369.

19. Léopold Sédar Senghor, «Les Leçons de Frobenius », préface à Leo Frobenius (1873-1973) : une anthologie, E. Haberland (dir.), Wiesbaden, Franz Steiner, 1973, p. VII-XIII, p. VII. Il évoque les circonstances de sa première lecture, dans les années 1930, à Paris. Voir également l'article qui porte le même titre que cette préface, mais en diffère «Les Leçons de Frobenius ", Présence africaine, 111, n³, 1978, p. 147-148.

20. André Breton, « Un grand poète noir » [1947], préface (puis postface) au Cahier d'un retour au pays natal, d'Aimé Césaire, Paris, Présence Africaine, 1983, p. 84.

21. Georges Balandier, Afrique ambiguë [1957], Paris, Plon, coll. "Terre humaine », 1985, p. 21. Comme Balandier le note aux débuts des années 2000, la génération d'écrivains qui succède à celle de la négritude va «rejet[er] Frobenius comme un colonialiste faisant sa cathédrale de 
l'Afrique, une cathédrale pour lui et pour le service du colonisateur », Georges Balandier, "Préface ", dans Marie-Claude Smouts (dir.), La Situation post-coloniale, Les post-colonial studies dans le débat français, Paris, Presses de Science Po, 2007, p. 21.

22. Dans Peau noire, masques blancs [1952], Paris, Seuil, coll. "Points essais », 2015, on lit par exemple : "pour nous, celui qui adore les nègres est aussi "malade" que celui qui les exècre ", p. 9. Pour une mise en regard du roman de Ouologuem et des problèmes discutés par Paulin Hountondji, voir Bachir Diagne «1968: crisis in African letters », Romanic Review, vol. 101, n 1-2, 2010, p. 149-159 ; Stanislas Adotevi, Négritude et négrologues, Paris, UGE, coll. «10/18 », 1972. La seconde partie, intitulée «Regard sur l'ethnologie», est une critique de l'ethnologie traditionnelle et de la négritude qui s'en fait la complice; sa thèse est la suivante : « La négritude, c'est la dernière-née d'une idéologie de domination. C'est l'aboutissement de plusieurs décades d'ethnologie [...] », « c'est la manière noire d'être blanc », p. 153 ; p. 207.

23. Yambo Ouologuem, Lettres à la France nègre, Paris, Nalis, 1969, p. 190-191.

24. "Saïf fabula et l'interprète traduisit, Madoubo répéta en français, raffinant les subtilités qui faisaient le bonheur de Shrobénius » (DV, p. 102).

25. «Une école africaniste ainsi accrochée aux nues du symbolisme magico-religieux, cosmologique et mythique, était née : tant et si bien que durant trois ans, des hommes - et quels hommes!: des fantoches, des aventuriers, des apprentis banquiers, des politiciens, des voyageurs, des conspirateurs, des chercheurs - "scientifiques", dit-on, en vérité sentinelles asservies, montant la garde devant le monument "shrobéniusologique" du pseudo-symbolisme nègre, accoururent au Nakem. » (DV, p. 112).

26. Comme le note Christopher Miller, «Ouologuem's response to false images of cultural identity is to undermine the notion of identity itself, to construct a universe in which all bonds of unity are bonds of enslavement, including the bond between Ouologuem's writing and the European texts that he plagiarized. Reading Le Devoir de violence contributes to the articulation between literature and anthropology only by showing how it has gone wrong in the past ", Theories of Africans, op. cit., p. 21

27. Dans sa «Lettre aux pisse-copies Nègres d'écrivains célèbres ", Ouologuem expose sa recette pour faire «un milliard de romans sans peine ", en invitant à des exercices de combinatoire. Il invite la « négraille » à s'emparer de « cette masse gigantesque d'œuvres des maîtres à penser qui puisent leur matière dans les archives du sang " et à faire du roman "une interminable perspective de références et possibilités de lecture ", Lettres à la France nègre, op. cit., p. 178.

28. Lettre citée par Jean-Pierre Orban, dans «Livre culte, livre maudit: Histoire du Devoir de violence de Yambo Ouologuem », op. cit.

29. De nombreux travaux ont été consacrés à cette intertextualité critique; voir notamment Joseph R. Slaughter, " "It is good to be primitive". African Allusion and the Modernist Fetish of Authenticity », Paul K. Saint-Amour (dir.), Modernism and Copyright, Oxford University Press, 2011, p. 275-301. L'auteur inscrit le débat suscité par le roman dans l'histoire politique de la propriété intellectuelle.

30. Voir sur ce point Michael Janis, Africa after Modernism: Transitions in Literature, Media, and Philosophy, New York, Routledge, 2008, chapitre «African Avant-Garde. Ouologuem's Anticolonialist Anthropology", p. 87-104; il voit à l'œuvre dans le roman une «(counter)ethnographic irony ». Le brouillage ironique joue aussi au niveau intratextuel. Dans les discours prêtés aux personnages, la source de l'énonciation peut ainsi troubler la compréhension de l'énoncé, même lorsque celui-ci semble clair. Ceci vaut par exemple pour le discours de résistance au colonialisme tenu par Saif, discours qui constate les effets de la décolonisation, alors qu'il est censément tenu au moment de la conquête (DV, p. 28). Il est peutêtre permis ici d'entendre la voix de l'auteur.

31. Sauf peut-être la figure de l'évêque Henry, même si la fin du roman (un dialogue entre Saïf et l'évêque autour d'une partie d'échecs) reste équivoque. 
32. Léopold Sédar Senghor, Les Fondements de l'africanité, Paris, Présence Africaine, 1967.

33. Thomas Hale, Scribe, Griot and Novelist, Narrative Interpreters of the Songhay Empire, op.cit., p. 139.

34. Le séjour en France de Kassoumi est marqué par une expérience d'aliénation affective dans une relation homosexuelle, récit qui n'est bien sûr pas étranger à la polémique suscitée par le roman.

35. Walter Benjamin, « Le caractère destructeur » [1931], E Euvres II, trad. fr. Rainer Rochlitz, Paris, Gallimard, coll. « Folio Essai », 2000, p. 332.

36. Bachir Diagne, « 1968 : crisis in African letters », op. cit., p. 154.

\section{RÉSUMÉS}

Cet article s'attache au personnage de Fritz Shrobénius, ethnologue de passage dans l'empire ouest-africain imaginaire du Nakem, dont Le Devoir de violence de Yambo Ouologuem (1968) est la chronique sanguinaire. Savant bouffon dont le nom est démarqué de celui de l'ethnologue allemand Leo Frobenius, ce personnage secondaire permet à l'auteur d'incriminer le primitivisme mercantile qui se met en scène sous des dehors scientifiques. Mais il lui permet aussi de marquer ses distances, par la relecture critique qu'il engage de l'œuvre de Frobenius, avec les partis anthropologiques des écrivains de la négritude, pour qui Frobenius était une référence centrale. Il constitue enfin une façon de faire voler en éclats, par les moyens de la fiction et les ressources de l'intertextualité, l'idée d'un sens unique - direction et signification - de la relation ethnographique.

This article focuses on the character of Fritz Shrobénius, an anthropologist who visits the imaginary west-African empire of Nakem, setting of Le Devoir de violence (Bound to violence), the bloodshed chronicle written by Yambo Ouologuem in 1968. This secondary character, a farcical scientist whose name is copied from the one of the German anthropologist Leo Frobenius, allows Ouologuem to incriminate the mercenary primitivism that uses science as a mask. Through the critical rereading of the work of Frobenius that he implies, the character also enables Ouologuem to distance himself from the anthropological bias of the negritude writers, for whom Frobenius was a major reference. The character is eventually a way to shatter, through the means of fiction and intertextuality, the conception of the ethnographic relation as a one-way and one-sensical process.

\section{INDEX}

Mots-clés : Yambo Ouologuem, Leo Frobenius, négritude, plagiat, ethnologie

\section{AUTEUR}

\section{ÉLÉONORE DEVEVEY}

Université de Genève 